\title{
Gadolinium-Enhanced Magnetic Resonance Imaging of Meningeal Carcinomatosis in Colon Cancer
}

\author{
Hiroyuki Kato, Sei Emura, Toshinobu Takashima, \\ Keizo Ohmori and Toshiaki Sunaga \\ Department of Internal Medicine, Saga Medical School, \\ Saga 849
}

Kato, H., Emura, S., Takashima, T., Ohmori, K. and Sunaga, T. Gadolinium-Enhanced Magnetic Resonance Imaging of Meningeal Carcinomatosis in Colon Cancer. Tohoku J. Exp. Med., 1995, 176 (2), 121-126 — Meningeal carcinomatosis has been infrequently reported in colorectal carcinoma. Recently the value of gadolinium-enhanced magnetic resonance imaging (MRI) in the examination of this disease has been described. We report a 55-year-old man with meningeal carcinomatosis from a known colon cancer. In this case, a gadoliniumenhanced MRI of the brain showed enhancing dura and nodular lesions which were not detected by enhanced computed tomography. The gadolinium-enhanced MRI may serve as an indicator of leptomeningeal involvement and may be a useful test in addition to the evaluation of cerebrospinal fluid cytology and chemistry. meningeal carcinomatosis; colon cancer; magnetic resonance imaging; computed tomography

Neoplastic involvement of the leptomeningeal membranes may occur as a metastatic process originating from a distant primary tumor. The most common meningeal tumor is acute lymphocytic leukemia. Meningeal metastases occur in a number of other malignancies: breast, lung, pancreas, stomach and prostate cancer, melanoma, and lymphoma (Posner 1971; Olson et al. 1974). However, meningeal carcinomatosis has been described infrequently in association with carcinoma of the colon (Arora 1973; Fisher and Weiss 1979; Kim et al. 1980; Smith et al. 1984). A recent article has found that contrast-enhanced magnetic resonance imaging (MRI) was more sensitive than contrast-enhanced computed tomography (CT) for the detection of meningeal carcinomatosis (Sze et al. 1989).

We report here the rare case of meningeal carcinomatosis in a patient with colon cancer who was diagnosed by cytology and gadolinium-enhanced MRI.

Received December 22, 1994; revision accepted for publication March 10, 1995.

Address for reprints: Hiroyuki Kato, Department of Internal Medicine, Saga Medical School, 5-1-1, Nabeshima, Saga 849, Japan. 


\section{Case Report}

A 55-year-old man was admitted to Saga Medical School Hospital on March 27, 1992, complaining of a headache, nausea, and vomiting for 4 days. Four months prior to this admission, he underwent a resection of adenocarcinoma of the descending colon. Extensive nodal involvement was found, and the tumor could not be completely excised. No liver metastases were seen at the time of laparotomy. Chemotherapy was not administered.

At this admission, his body temperature was $36.3^{\circ} \mathrm{C}$, and he had a blood pressure of $154 / 70 \mathrm{mmHg}$ with a pluse rate of 60 beats $/ \mathrm{min}$. He was fully conscious and oriented. A neurological examination revealed no neck stiffness and no focal neurological sign. Papilledema was not present. A surgical scar was noted in his abdomen. There was no regional lymphadenopathy. The rectal examination was negative for blood. The rest of his physical examination was noncontributory.

His initial laboratory findings included the white blood cell count of 5,300/ $\mu 1$ with a normal differential, the erythrocyte count of $425 \times 10^{4} / \mu 1$, the hemoglobin concentration of $11.5 \mathrm{~g} / 100 \mathrm{ml}$, the hematocrit of $35.6 \%$, the platelet count of $34.5 \times 10^{4} / \mu \mathrm{l}$; the serum glutamic oxaloacetic transaminase value of $16 \mathrm{IU} /$ liter

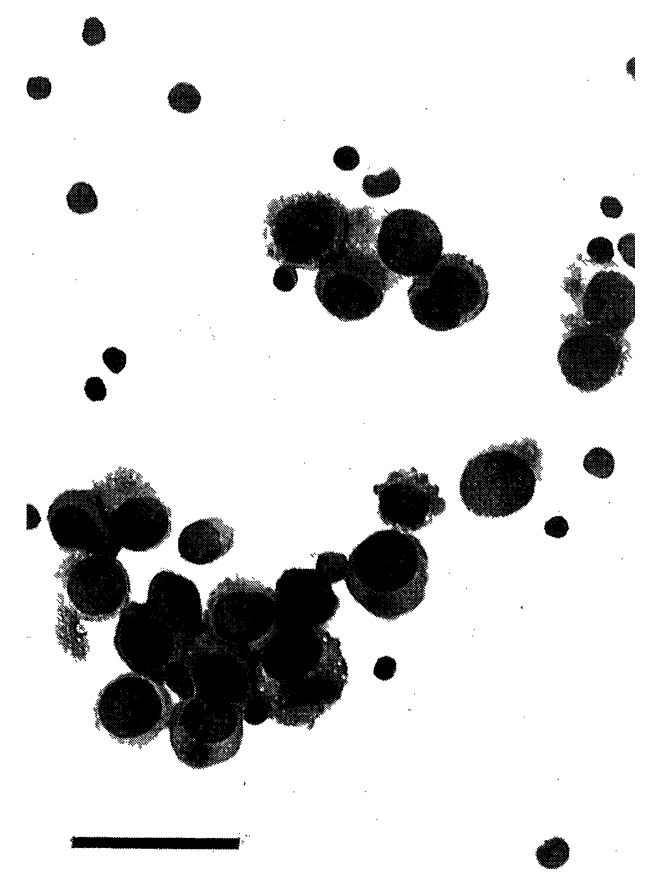

Fig. 1. CSF cytology on admission. The clustered cells have large, ovoid nuclei with prominent nucleoli (Giemsa stain, bar: $40 \mu \mathrm{m}$ ). 
(normal, 10-35 IU/liter), the serum glutamic pyruvic transaminase value of 14 IU/liter (normal, 0-40 IU/liter), the serum lactate dehydrogenase of $308 \mathrm{IU} /$ liter (normal, 200-450 IU/liter), the blood urea nitrogen of $19.0 \mathrm{mg} / 100 \mathrm{ml}$, and the serum creatinine of $0.52 \mathrm{mg} / 100 \mathrm{ml}$. His erythrocyte sedimentation rate was 13
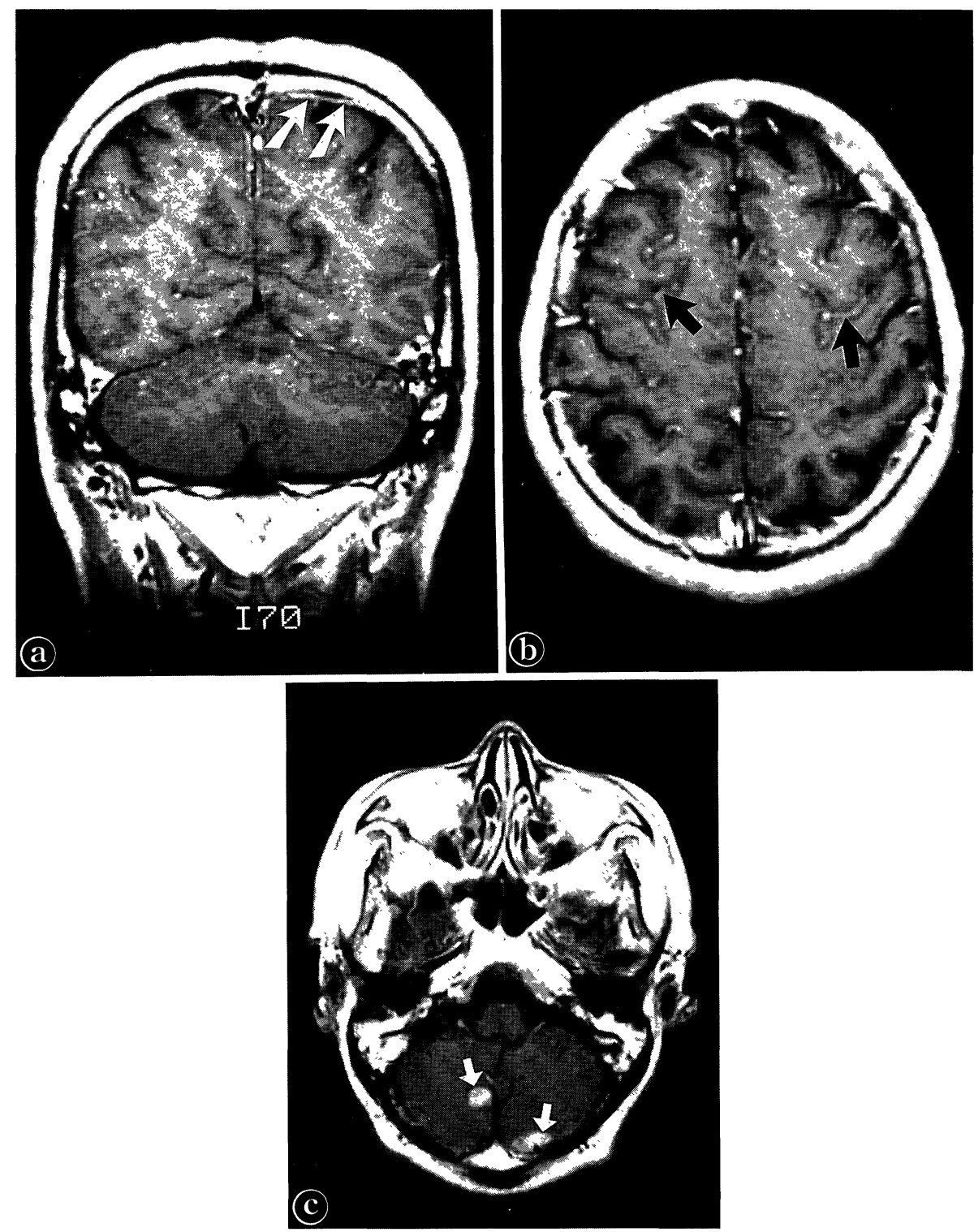

Fig. 2. Gadolinium-enhanced MRI of the brain. a: Frontal section. The dura was enhanced in the cerebral hemispheres (arrows). b: Horizontal section. The dura was enhanced in the cortical sulci (arrows). c: Horizontal section. Enhancing nodular lesions are noted in the cerebellar hemispheres and vermis cerebelli (arrows). 
$\mathrm{mm} /$ first hour. His urine analysis was normal. A chest $\mathrm{x}$-ray detected no metastatic lesions in the lung. Skull films were negative. An x-ray of the abdomen demonstrated no signs of ileus. The electrocardiogram was within normal limits. Computed tomographies (plain and enhanced) of the brain did not reveal any mass lesions. A lumbar puncture revealed an opening cerebrospinal fluid (CSF) pressure of $280 \mathrm{mmH}_{2} \mathrm{O}$ with clear, colorless fluid, the protein concentration of $34 \mathrm{mg} / 100 \mathrm{ml}$, and the glucose concentration of $55 \mathrm{mg} /$ $100 \mathrm{ml}$ (with the peripheral glucose level of $123 \mathrm{mg} / 100 \mathrm{ml}$ ). The CSF sample contained no red cells, 34 lymphocytes, and 108 abnormal cells per $\mathrm{mm}^{3}$. These abnormal cells had large, ovoid nuclei with prominent nucleoli and intracytoplasmic mucin, and formed clusters (Fig. 1). These cells were thought to be consistent with an adenocarcinoma of gastrointestinal origin. The carcinoembryonic antigen (CEA) level in CSF was $10.2 \mathrm{ng} / \mathrm{ml}$ while the plasma CEA level $15.9 \mathrm{ng} /$ $\mathrm{ml}$ (normal, less than $5 \mathrm{ng} / \mathrm{ml}$ ). CSF cultures were negative. A gadoliniumenhanced MRI demonstrated enhancement of the dura of cerebral hemisphere (Fig. 2a) and the cortical sulci (Fig. 2b). Multiple enhancing nodular lesions (less than $10 \mathrm{~mm}$ in diameter) were noted in the cerebellar hemispheres and vermis cerebelli (Fig. 2c). The patient was treated with intrathecal methotrexate (15 $\mathrm{mg})$, cytosine arabinoside $(40 \mathrm{mg})$, and prednisolone $(20 \mathrm{mg})$, followed by whole brain radiation (100 cGy/day). His headache and nausea improved transiently, but he lapsed into a coma and died 5 days after beginning of radiation treatments. The post-mortem was not obtained.

\section{Discussion}

Colorectal carcinoma rarely metastasize to the central nervous system (CNS), and metastatic involvement of the leptomeninges is an even more uncommon event. A review of the literature (MEDLINE; National Library of Medicine, Bethesda, MD, USA) yielded only four reported cases of meningeal carcinomatosis secondary to colorectal carcinoma (Arora 1973; Fisher and Weiss 1979; Kim et al. 1980; Smith et al. 1984). Examination of the CSF is of the utmost importance in making the diagnosis of meningeal carcinomatosis (Glass et al. 1979). The CT may be helpful in the diagnosis of this disease but is sometimes falsely negative (Theodore and Gendelman 1981; Wasserstrom et al. 1982). In our case, an enhanced CT of the brain was negative while the CSF cytology was positive. Recently, MRI is becoming an acceptable alternative imaging modality for the detection, characterization, and follow-up of numerous disease in the CNS. However, unenhanced MRI should not replace CT as the initial diagnostic evaluation for the leptomeningel progression of a tumor, since it may also be falsely negative if it is used without enhancement (Davis et al. 1987; Krol et al. 1988). On the other hand, a recent article has indicated the value of gadolinium enhanced MRI in the diagnosis of intraspinal metastasis (Fredericks et al. 1989; Parizel et al. 1989), bacterial meningitis (Mathews et al. 1988; Chang et al. 1990), and 
meningeal carcinomatosis (Sze et al. 1989; Rodesch et al. 1990). The enhanced $\mathrm{MRI}$ is more sensitive than contrast-enhanced $\mathrm{CT}$ in the examination of meningeal disease because gadolinium may be more sensitive in delineating blood-brain barrier abnormalities than iodinated contrast (Mathews et al. 1988). This advantage is especially beneficial in areas adjacent to cortical bone where contrast enhancement on CT may be obscured (Mathews et al. 1988). The abnormal findings of meningeal carcinomatosis detected by gadolinium-enhanced MRI include sharp contrast uptake in the leptomeningeal areas and nodular deposits (Sze et al. 1989; Rodesch et al. 1990). In this case, a gadolinium-enhanced MRI of the brain showed enhancing dura and nodular lesions which were not detected by $\mathrm{CT}$.

The current treatment of this disease includes intrathecal chemotherapy and CNS irradiation (Seeldrayers and Hildebrand 1984). In advanced stages of carcinomatosis, an assessment of the effectiveness of treatment by serial lumbar punctures may be difficult to perform because of the presence of intracranial hypertension. In such cases, enhanced MRI might provide a useful method for evaluating the efficacy of treatment.

In summary, we have reported the rare case of meningeal carcinomatosis in colon cancer patient who had significant findings on an enhanced MRI of the brain. Any patient with known colorectal carcinoma who develops neurological symptoms in the presence of a normal CT of the brain should be evaluated carefully for meningeal carcinomatosis. In addition to an examination of the CSF cytology and chemistries, a gadolinium-enhanced MRI may serve as an indicator of leptomeningel involvement.

\section{References}

1) Arora, Y.R. (1973) Colonic carcinoma presenting with meningeal metastases. J.R. Coll. Surg. Edinb., 18, 376-378.

2) Chang, K.H., Han, M.H., Roh, J.K., Kim, I.O., Han, M.C. \& Kim, C.W. (1990) Gd-DTPA-enhanced MR imaging of the brain in patients with meningitis: Comparison with CT. Am. J. Neuroradiol., 11, 69-76.

3) Davis, P.C., Friedman, N.C., Fry, S.M., Malko, J.A., Hoffmann, J.C., Jr. \& Braun, I.F. (1987) Leptomeningeal metastasis: MR imaging. Radiology, 163, 449-454.

4) Fisher, M.A. \& Weiss, R.B. (1979) Carcinomatous meningitis in gastrointestinal malignancies. South. Med. J., 72, 930-932.

5) Fredericks, R.K., Elster, A. \& Walker, F.O. (1989) Gadolinium-enhanced MRI: A superior technique for the diagnosis of intraspinal metastases. Neurology, 39, 734-736.

6) Glass, J.P., Melamed, M., Chernik, N.L. \& Posner, J.B. (1979) Malignant cells in cerebrospinal fluid (CSF): The meaning of a positive CSF cytology. Neurology, 29, 1369-1375.

7) Kim, K.S., Weinberg, P.E. \& Hemmati, M. (1980) Spinal pachymeningeal carcinomatosis; Myelographic features. Am. J. Neuroradiol., 1, 199-200.

8) Krol, G., Sze, G., Malkin, M. \& Walker, R. (1988) MR of cranial and spinal meningeal carcinomatosis: Comparison with CT and myelography. Am. J. Neuroradiol., 9, 709-714. 
9) Mathews, V.P., Kuharik, M.A., Edwards, M.K., D'Amour, P.G., Azzarelli, B. \& Dreesen, R.G. (1988) Gd-DTPA-enhanced MR imaging of experimental bacterial meningitis: Evaluation and comparison with CT. Am. J. Neuroradiol., 9, 1045-1050.

10) Olson, M.E., Chernik, N.L. \& Posner, J.B. (1974) Infiltration of the leptomeninges by systemic cancer. A clinical and pathologic study. Arch. Neurol., 30, 122-137.

11) Parizel, P.M., Baleriaux, D., Rodesch, G., Segebarth, C., Lalmand, B., Christophe, C., Lemort, M., Haesendonck, P., Niendorf, H.P., Flament-Durand, J. \& Brotchi, J. (1989) Gd-DTPA-enhanced MR imaging of spinal tumors. Am. J. Neuroradiol., 10, 249-258.

12) Posner, J.B. (1971) Neurological complications of systemic cancer. Med. Clin. North. Am., 55, 625-646.

13) Rodesch, G., Bogaert, P.V., Mavroudakis, N., Parizel, P.M., Martin, J.J., Segebarth, C., Vyve, M.V., Baleriaux, D.\& Hildebrand, J. (1990) Neuroradiologic findings in leptomeningeal carcinomatosis: The value interest of gadolinium-enhanced MRI. Neuroradiology, 32, 26-32.

14) Seeldrayers, P. \& Hildebrand, J. (1984) Treatment of neoplastic meningitis. Eur. J. Cancer. Clin. Oncol., 20, 449-456.

15) Smith, R.S., Zatzkin, J.B. \& Hynes, H.E. (1984) Carcinomatous meningitis. $J$. Kans. Med. Soc., 85, 9-10.

16) Sze, G., Soletsky, S., Bronen, R.\& Krol, G. (1989) MR imaging of the cranial meninges with emphasis on contrast enhancement and meningeal carcinomatosis. Am. J. Neuroradiol, 10, 965-975.

17) Theodore, W.H. \& Gendelman, S. (1981) Meningeal carcinomatosis. Arch. Neurol., 38, 696-699.

18) Wasserstrom, W.R., Glass, J.P. \& Posner, J.B. (1982) Diagnosis and treatmnet leptomeningeal metastases from solid tumors: Experience with 90 patients. Cancer, 49, 759-772. 